\title{
Roux en Y Gastric Bypass: How and Why it Fails?
}

Mark A Dykstra ${ }^{1}$, Noah J Switzer ${ }^{*}$, Vadim Sherman ${ }^{2}$, Shahzeer Karmali ${ }^{1,3}$ and Daniel W Birch ${ }^{1,3}$

${ }^{1}$ Department of Surgery, University of Alberta, Edmonton, Alberta, Canada

${ }^{2}$ Department of Surgery, Houston Methodist Hospital, Houston, Texas, USA

${ }^{3}$ Center for the Advancement of Minimally Invasive Surgery (CAMIS), Royal Alexandria Hospital, Edmonton, Alberta, Canada

\begin{abstract}
The Roux-en-Y Gastric Bypass (RYGB) is the most successful bariatric procedure. Despite its successes, a growing number of patients who undergo RYGB present with clinically significant weight regain in the years following their procedure. Anatomical etiologies have been often implicated in this weight regain as either an enlargement of the gastric pouch or gastrojejunostomy, or the presence of a gastro gastric fistula leading to loss of caloric restriction. Surgical or endoscopic revision is an effective means to address this. Behavioral problems can also lead to poor results if patients do not adhere to strict dietary and lifestyle regimens following their procedure. Poor dietary compliance needs to be addressed with behavioral and nutritional counseling at a multidisciplinary clinic. Mental health is an often forgotten etiology for weight recidivism. Especially in high-risk patients - patients with personality disorders and addictions - simply recognizing the possibility of mental health problems during a preoperative assessment is a good start. Lastly, gut hormone imbalances including ghrelin and insulin can lead separately to increased appetite and significant hypoglycemia respectively, which can theoretically lead to RYBG failure. However, more research needs to be devoted to this area in order to be fully comfortable with making a conclusion. Overall, successful strategies for the management and recognition of weight recidivism following RYGB is important as these patients make up an important and growing segment of any bariatric practice.
\end{abstract}

Keywords: Roux en Y Gastric Bypass (RYGB); Gastrojejunostomy

\section{Introduction}

The Roux en Y Gastric Bypass (RYGB) is the most studied and performed bariatric procedure in North America today [1]. It is widely considered the gold standard in bariatric surgery; achieving superior weight-loss with acceptable complication rates. Furthermore, patients undergoing RYGB has experienced significant improvement and possibly resolution of diabetes mellitus, hypertension, dyslipidemia and sleep apnea, along with a subsequent reduction in overall mortality $[2,3]$. However, despite the successful outcomes of RYGB, weight recidivism is becoming a significant issue, with a significant amount of patients shown to re-gain weight in the years following their surgery [4]. The consequences of this weight regain are significant both to the patient, namely the redevelopment of obesity related co-morbidities, and to the health care system, as the economic ramifications of obesity are costly.

The RYGB involves the creation of a small proximal gastric pouch and altered intestinal continuity. Two convergent limbs of small bowel are created; an alimentary or Roux limb, which is directly connected to the gastric pouch, and a biliopancreatic limb, containing secretions from the excluded stomach, gallbladder and pancreas. The Roux limb is generally between $100-150 \mathrm{~cm}$ and the biliopancreatic limb includes the duodenum and $30 \mathrm{cms}$ of the proximal jejunum. The RYGB configuration is particularly effective as it results in both malabsorptive and restrictive weight-loss, thereby both limiting oral intake and caloric absorption.1 The unique anatomy of the RYGB makes it less susceptible to weight regain, compared to other procedures that only offer restriction. In this paper, we review the literature for failures of the Roux-en-Y Gastric Bypass due to weight recidivism, theorize why weight regain can occur and recommend strategies for the prevention and management of this complication.

\section{Discussion}

No primary bariatric procedure is immune from some percentage of weight regain. While malabsorptive surgeries are less susceptible compared to purely restrictive procedures, significant weight gain, described as an increase in body weight of $10 \mathrm{lbs}$ from nadir, occurs in $10-20 \%$ of RYGB patients potentially equating to a failure of the procedure $[2,5]$. Furthermore, longer term studies have shown that at ten-year follow up RYGB failure rates, defined as a Body Mass Index (BMI) $>35$ or \% Excess Weight Loss (EWL) less than $50 \%$, was between 15 to $35 \%[6,7]$. It appears, not unexpectedly, that weight regain increases steadily in the years following RYGB, as the majority of patients report some weight regain from their nadir weight, with the super-obese population, defined as a BMI $\geq 50 \mathrm{~kg} / \mathrm{m}^{2}$, being especially at risk $[4,8]$.

\section{Theories to explain weight recidivism following RYGB}

Possible explanations for this significant weight recidivism following RYGB fall under four main categories: anatomic, behavioral, psychological, and hormonal/metabolic. Often the reasons are multifactorial, as affected individuals will have elements from all four categories.

\section{Anatomical}

Enlargement of the gastric pouch and gastrojejunostomy (stoma) are important anatomic considerations for assessing weight recidivism following RYGB. Recent studies by Heneghan et al. and Yimcharoen et al. Looking at weight regain following RYGB both found that over $70 \%$ of patients who experienced post-operative weight recidivism had abnormal anatomy. This amounted to either an enlarged gastrojejunostomy diameter and/or enlarged gastric pouch, which was significantly greater than their control patients [2,5]. Enlargement of the gastric pouch and stoma may lead to loss of restriction, an important mechanism of weight loss in RYGB.

*Corresponding author: Noah Switzer, Department of Surgery, University of Alberta, Edmonton, Alberta, Canada, 8440112 St NW, Edmonton, AB T6G 2P4, Canada, Tel: (780) 884-2964; Fax: (780) 735-6652; E-mail: nswitzer@ualberta.ca

Received January 03, 2014; Accepted January 28, 2014; Published February 05, 2014

Citation: Dykstra MA, Switzer NJ, Sherman V, Karmali S, Birch DW (2014) Roux en Y Gastric Bypass: How and Why it Fails? Surgery Curr Res 4: 165 doi:10.4172/2161-1076.1000165

Copyright: (c) 2014 Dykstra MA, et al. This is an open-access article distributed under the terms of the Creative Commons Attribution License, which permits unrestricted use, distribution, and reproduction in any medium, provided the original author and source are credited. 
A second anatomical complication that can lead to weight recidivism post-RYGB is the formation of a gastro-gastric fistula. First described in 1995, gastro gastric fistulas are abnormal communications between the surgically created gastric pouch and the excluded stomach [9]. The fistula can negate the restrictive aspect of RYGB, and allow the patient to ingest larger volumes of food without the sensation of early satiety, leading to post-operative weight gain [10]. Gastro gastric fistulas are more commonly seen in the setting of a non-partioned gastric bypass, where the pouch and excluded stomach are separated by an uncut staple line. With the advent of new stapling techniques, where the pouch and excluded stomach are physically cut and separated, the incidence of these fistulas has decreased. Although this is a rare complication, occurring in 15 of 1292 (1.2\%) of consecutive patients who underwent RYGB in a study by Carrodeguas et al., gastro gastric fistula should be ruled out as a cause when working up patients with post RYGB weight recidivism [11].

\section{Strategies to address Anatomic issues}

If a patient has regained weight following a RYGB, it is reasonable to look for and treat anatomical abnormalities. If an enlarged gastric pouch or stoma is identified, numerous endoscopic interventions have been described. Endoscopic procedures are generally considered safe procedures that carry little patient morbidity and are cost effective [12]. Catalano et al. found that if the etiology of weight regain was due to stomal dilation that the endoscopic injection of a sclerosant (sodium morrhuate) led to a reduction in both stoma size and in weight regain in $64 \%$ of patients [13]. A pilot study looking at endoscopic suturing to reduce stoma size resulted in an average weight loss of $23.4 \%$ of excess body weight [14]. Goyal et al. propose recreating restriction using gastric plication with the endoscopic tool StomaphyX to reduce the gastric pouch and stoma [12]. Unfortunately, one of the biggest limitations is that there are a paucity of studies comparing the pouch anatomy of RYGB with weight regain versus those without, thereby making it difficult to ascribe the reason for weight regain to an anatomical factor such as stomal or pouch enlargement.

The main treatment for anatomical failures leading to weight regain is revisional bariatric surgery, involving adjustable gastric banding, conversion to biliopancreatic diversion-duodenal switch, and conversion to a distal-RYGB [15]. However, the risk of complication of a revisional procedure is higher compared with the primary bariatric procedures and the long-term success of these revisional procedures has yet to be fully elucidated [16].

Revisional procedures to manage anatomic failure after RYGB have been performed for decades. Most procedures entail surgical re-creation of the pouch or stoma using either suture or stapling devices. Unfortunately, these procedures are fraught with extremely high complication and mortality rates [5]. There are three surgical strategies to manage weight recidivism post Roux en $Y$ gastric bypass. The first option entails application of an adjustable gastric band to the RYGB. This procedure entails placing a LAGB on the gastric pouch of the RYGB in an attempt to promote greater gastric restriction for the patient. A systematic review by Vijgen et al, seven studies with a total of 94 patients reported further weight loss, varying from 55.9\%-94.2\% excess body mass index loss (EBMIL) after 12-42 months of follow-up [17].

The second surgical option involves increasing the malabsorptive effect of the RYGB by lengthening the roux limb. This procedure converts a proximal (conventional) Roux-en-Y gastric bypass to a distal Roux-en-Y gastric bypass. It is usually only considered when the patient has a very low base metabolic rate and continues with poor weight loss results despite an augmented exercise program. Studies examining this option are limited. Evidence from Christou et al. found that at 5 years there was no difference in BMIs in RYGB patients who had "shorter" Roux limbs versus "longer" limbs [6]. Bowel pliability and adaptation may counteract the intended surgically created malabsorption and temper weight loss. As such this option is not a popular option among bariatric surgeons managing weight regain post RYGB. The third, and most popular option in revising a RYGB, involves conversion of the RYGB to a duodenal switch. A study by Parikh et al. evaluated 12 patients undergoing a conversion from Roux-en-Y gastric bypass to a duodenal switch. The patients had an average original BMI of 53.9, an average low BMI after gastric bypass of 31.6 and an average preconversion BMI of 40.7) [18]. Patients lost a dramatic amount of weight after conversion to biliopancreatic diversion with duodenal switch (BPD-DS), with a mean body mass index and excess weight loss of 31 $\mathrm{kg} / \mathrm{m}(2)$ and $63 \%$, respectively, at 11 months postoperatively. Other types of revisional therapy after failed RYGB include reversal to native anatomy with or without a sleeve gastrecomy, however this procedure carries a significant leak rate, with $30 \%$ of patients suffering a leak, and thus is not favoured over the BPD-DS [16]. All co-morbidities resolved completely with the weight loss [18]. It is important to recognize that patient selection is maybe the most important consideration prior to considering the risks of a re-operation and this is best assessed at a dedicated weight recidivism clinic consisting of surgeons along with nurses, psychologists and nutritionists.

\section{Behavioral}

Failure to adhere to dietary recommendations following RYGB is a major reason for failure $[8,19,20]$. Not surprisingly, patients who consume a high caloric diet are found to be at increased risk for weight regain following RYGB.8 In a survey of 208 patients who underwent RYGB, Odom et al. identified insuppressible food urges as a major predictor of weight regain [20]. This insuppressible food urge leads to an increased intake of calories, snacks, sweets and fatty foods, which make maintenance of prominent post-surgical weight loss difficult.8 These foods carry a high calorie density, indicating that although the patients do not necessarily ingest a large amount, they incur a significant surplus of caloric intake. In a similar study, Kofman et al. identified two other maladaptive eating behaviors, binge eating and grazing, that are independent predictors of increased weight regain following gastric bypass [19].

In addition to poor eating habits, Freire et al. looked at physical inactivity as a predictive tool in weight regain following RYGB. They reported that patients who were regularly physically active reported less weight regain than those who were not regularly active [8].

\section{Strategies to address behavioral issues}

Addressing patient behavior is important when trying to prevent and treat RYGB failures. Continued post-operative follow up from a multi-disciplinary team including dieticians, psychologists, nurses and surgeons has been shown improve patient compliance and lead to impressive EWL [21]. In fact, patients without post-operative follow up are 4.6 times more likely to experience weight recidivism compared to patients who had 4-5 visits a year [20]. A meta-analysis of behavioral management following bariatric surgery reported a significant association between support group attendance and postoperative weight loss [22]. Furthermore, in respect to nutritional counseling, Freire et al. found that although $85.3 \%$ of patients attended nutritional counseling in the first two years post-op, only $3 \%$ were still attending 5 years after the RYGB and this lack of dietary follow up leads to poor results [8]. Patients also need to take an active role and be proactive 
in the management of their weight loss regimen. It has been shown that self-monitoring, including keeping updated food records and performing regular weight checks, has a positive association with the maintenance of weight loss [20].

\section{Psychological}

The mental health of bariatric patients must also be considered when looking at weight recidivism. In a study of 60 veterans, Rutledge et al. found that patients with 2 or more psychiatric conditions were 6.4 times more likely to have cessation of weight loss or weight gain one year after bariatric surgery when compared to patients with one or no psychiatric conditions [23]. Interestingly, patients with a binge eating disorder gained back almost double the weight (13.9 lbs compared to $7.1 \mathrm{lbs}$ ) from their minimum weight post-RYGB compared to nonbinge eaters [24]. Although a meta-analysis looking for preoperative predictors of weight loss did not find a relationship between a preoperative diagnosis of binge eating and post-surgical weight change, best practices for the behavioral and psychological care of bariatric surgery candidates includes a preoperative assessment for binge eating $[25,26]$. Other psychological predictors of weight gain following RYGB surgery include personality disorders, depression, and alcohol and drug use $[20,25]$.

\section{Strategies to address Psychologic issues}

Identifying psychiatric conditions in patients who have had weight recidivism following RYGB is an important factor in helping to explain why the RYGB has failed and should be implemented in preoperative assessments [26]. Patients who are found to have mal-adaptive eating habits should be referred to behavioral therapy. There is evidence in the literature that post-operative intervention may be more successful than pre-operative intervention for behavioral modification. Leahey et al. reported that patients were significantly more likely to complete behavioral intervention (91\% versus 14\%) after the RYGB has occurred rather than prior to the operation [27].

\section{Hormonal/Metabolic}

Gut hormone regulation is increasingly recognized as an important mechanism of action for malabsorptive bariatric procedures. Ghrelin in particular is an appetite-stimulating hormone whose pathologic regulation is evident in morbidly obese individuals. After RYGB, ghrelin levels are markedly reduced, especially post-prandially, leading in part to the successful weight loss in the short term following RYGB $[28,29]$. A recent study showed that long term levels of ghrelin slowly increase which potentially could correlate to weight regain, however this inference is debated as other groups have reported no difference between weight gain and pre or post-prandial ghrelin levels [30,31].

Peptide YY (PYY) is a satiety controlling gut hormone that is another regulator of the gut-brain axis. It has been shown that following RYGB, the post prandial release of this hormone is exaggerated in patients with good weight-loss following surgery leading to the cessation of the hunger drive, but is blunted in patients with suboptimal results [32].

Reactive hypoglycemia has also been implicated in post-RYGB as a source for weight recidivism. A study by Roslin et al. found that $91 \%$ (10/11) of the RYGB patients who had gained back greater than $10 \%$ of their initial weight loss had hypoglycemia after a 4-hour glucose tolerance test [33]. Glucose is an important regulator of appetite, and the anatomical changes following RYGB result in shortened gastric transit time of simple carbohydrates. The authors propose that the shortened transit time causes a surge of insulin post-prandially, which leads to reactive hypoglycemia. It is this hypoglycemia that is believed to stimulate hunger just a few hours after meals. Patients with reactive hypoglycemia are at increased risk for grazing and an overall increase in caloric intake [33].

\section{Strategies to address Hormonal/Metabolic issues}

Both the roles of Ghrelin and PYY in long-term weight regain need to be further clarified. Therefore, it is too early to address management strategies in dealing with these important gut hormones. To manage patients that have gained weight post RYGB and are consistently hypoglycemic post-prandially, Roslin et al. suggest that patients eat foods with low glycemic indexes, do not go too long between meals, and add bulk to their food [33]. The aim of this diet is to avoid the fluctuations in blood glucose levels and thereby reduce the appetite stimulation caused by hypoglycemia.

\section{Conclusion}

Although RYGB has proven to be a very effective treatment for obesity and its related co-morbidities, a certain percentage of patients will present with failure. Often the reasons for failure are multifactorial, and these patients need to be evaluated for anatomic, behavioral, psychological, and hormonal/metabolic, reasons for their weight regain. Working with a multidisciplinary team to evaluate eating habits, mental health, metabolic abnormalities, and post-surgical anatomy should provide the bariatric team with options to treat the recidivism and improve the quality of life for failed RYGB patients.

\section{References}

1. Khwaja H, Bonanomi G (2010) Bariatric surgery: Techniques, outcomes and complications. Current Anaesthesia and Critical Care 21: 31-38.

2. Heneghan HM, Yimcharoen P, Brethauer SA, Kroh M, Chand B (2012) Influence of pouch and stoma size on weight loss after gastric bypass. Surg Obes Relat Dis 8: 408-415.

3. Buchwald H, Avidor $\mathrm{Y}$, Braunwald E, Jensen MD, Pories W, et al. (2004) Bariatric surgery: a systematic review and meta-analysis. JAMA 292: 17241737.

4. Magro DO, Geloneze B, Delfini R, Pareja BC, Callejas F, et al. (2008) Long term weight regain after gastric bypass: a 5 -year prospective study. Obes Surg 18: 648-651.

5. Yimcharoen $P$, Heneghan HM, Singh M, Brethauer S, Schauer $P$, et al. (2011) Endoscopic findings and outcomes of revisional procedures for patients with weight recidivism after gastric bypass. Surg Endosc 25: 3345-3352.

6. Christou NV, Look D, Maclean LD (2006) Weight gain after short- and long-limb gastric bypass in patients followed for longer than 10 years. Ann Surg 244 734-740.

7. Valezi AC, de Almeida Menezes M, Mali J Jr (2013) Weight loss outcome afte Roux-en-Y gastric bypass: 10 years of follow-up. Obes Surg 23: 1290-1293.

8. Freire RH, Borges MC, Alvarez-Leite JI, Toulson Davisson Correia MI (2012) Food quality, physical activity, and nutritional follow-up as determinant of weight regain after Roux-en-Y gastric bypass. Nutrition 28: 53-58.

9. Cucchi SG, Pories WJ, MacDonald KG, Morgan EJ (1995) Gastrogastric fistulas. A complication of divided gastric bypass surgery. Ann Surg 221: $387-$ 391.

10. Filho AJ, Kondo W, Nassif LS, Garcia MJ, Tirapelle Rde A, et al. (2006) Gastrogastric fistula: a possible complication of Roux-en-Y gastric bypass. JSLS 10: 326-331.

11. Carrodeguas L, Szomstein S, Soto F, Whipple O, Simpfendorfer C, et al. (2005) Management of gastrogastric fistulas after divided Roux-en-Y gastric bypass surgery for morbid obesity: analysis of 1,292 consecutive patients and review of literature. Surg Obes Relat Dis 1: 467-474.

12. Goyal V, Holover S, Garber S (2013) Gastric pouch reduction using StomaphyX in post Roux-en- $Y$ gastric bypass patients does not result in sustained weight loss: a retrospective analysis. Surg Endosc 27: 3417-3420.

13. Catalano MF, Rudic G, Anderson AJ, Chua TY (2007) Weight gain after bariatric 
surgery as a result of a large gastric stoma: endotherapy with sodium morrhuate may prevent the need for surgical revision. Gastrointest Endosc 66: 240-245.

14. Thompson CC, Slattery J, Bundga ME, Lautz DB (2006) Peroral endoscopic reduction of dilated gastrojejunal anastomosis after Roux-en-Y gastric bypass: a possible new option for patients with weight regain. Surg Endosc 20: 17441748.

15. Rawlins ML, Teel D 2nd, Hedgcorth K, Maguire JP (2011) Revision of Rouxen-Y gastric bypass to distal bypass for failed weight loss. Surg Obes Relat Dis 7: $45-49$.

16. Vilallonga $R$, van de Vrande S, Himpens J (2013) Laparoscopic reversa of Roux-en-Y gastric bypass into normal anatomy with or without sleeve gastrectomy. Surg Endosc 27: 4640-4648.

17. Vijgen GH, Schouten R, Bouvy ND, Greve JW (2012) Salvage banding for failed Roux-en-Y gastric bypass. Surg Obes Relat Dis 8: 803-808.

18. Parikh M, Pomp A, Gagner M (2007) Laparoscopic conversion of failed gastric bypass to duodenal switch: technical considerations and preliminary outcomes. Surg Obes Relat Dis 3: 611-618.

19. Kofman MD, Lent MR, Swencionis C (2010) Maladaptive eating patterns, quality of life, and weight outcomes following gastric bypass: results of an Internet survey. Obesity (Silver Spring) 18: 1938-1943.

20. Odom J, Zalesin KC, Washington TL, Miller WW, Hakmeh B, et al. (2010) Behavioral predictors of weight regain after bariatric surgery. Obes Surg 20 : 349-356.

21. Gill RS, Switzer N, Driedger M, Shi X, Vizhul A, et al. (2012) Laparoscopic sleeve gastrectomy with staple line buttress reinforcement in 116 consecutive morbidly obese patients. Obes Surg 22: 560-564

22. Rudolph A, Hilbert A (2013) Post-operative behavioural management in bariatric surgery: a systematic review and meta-analysis of randomized controlled trials. Obes Rev 14: 292-302

23. Rutledge T, Groesz LM, Savu M (2011) Psychiatric factors and weight loss patterns following gastric bypass surgery in a veteran population. Obes Surg 21: 29-35.
24. Kalarchian MA, Marcus MD, Wilson GT, Labouvie EW, Brolin RE, et al. (2002) Binge eating among gastric bypass patients at long-term follow-up. Obes Surg 12: $270-275$

25. Livhits M, Mercado C, Yermilov I, Parikh JA, Dutson E, et al. (2012) Preoperative predictors of weight loss following bariatric surgery: systematic review. Obes Surg 22: 70-89.

26. Greenberg I, Sogg S, M Perna F (2009) Behavioral and psychological care in weight loss surgery: best practice update. Obesity (Silver Spring) 17: 880-884.

27. Leahey TM, Bond DS, Irwin SR, Crowther JH, Wing RR (2009) When is the best time to deliver behavioral intervention to bariatric surgery patients: before or after surgery? Surg Obes Relat Dis 5: 99-102.

28. Engström BE, Ohrvall M, Sundbom M, Lind L, Karlsson FA (2007) Mea suppression of circulating ghrelin is normalized in obese individuals following gastric bypass surgery. Int J Obes (Lond) 31: 476-480.

29. Geloneze B, Tambascia MA, Pilla VF, Geloneze SR, Repetto EM, et al. (2003) Ghrelin: a gut-brain hormone: effect of gastric bypass surgery. Obes Surg 13: $17-22$

30. Terra X, Auguet T, Guiu-Jurado E, Berlanga A, Orellana-Gavaldà JM, et al (2013) Long-term changes in leptin, chemerin and ghrelin levels following different bariatric surgery procedures: Roux-en-Y gastric bypass and sleeve gastrectomy. Obes Surg 23: 1790-1798.

31. Christou NV, Look D, McLean AP (2005) Pre- and post-prandial plasma ghrelin levels do not correlate with satiety or failure to achieve a successful outcome after Roux-en-Y gastric bypass. Obes Surg 15: 1017-1023.

32. Pournaras DJ, Osborne A, Hawkins SC, Mahon D, Ghatei MA, et al. (2010) The gut hormone response following Roux-en-Y gastric bypass: cross-sectional and prospective study. Obes Surg 20: 56-60.

33. Roslin M, Damani T, Oren J, Andrews R, Yatco E, et al. (2011) Abnormal glucose tolerance testing following gastric bypass demonstrates reactive hypoglycemia. Surg Endosc 25: 1926-1932. 